\title{
Comments on "Overviews of Models Defined with Charts of Concepts" by X. Castellani
}

\author{
Zheying Zhang \\ Department of Computer Science and Information Systems, University of Jyväskylä \\ P. O. Box 35, 40351 Jyväskylä, Finland \\ Email: zhezhan@.cc.jvu.fi
}

This paper has introduced a simplified model for the representation of system development methods. The model forms charts of concepts. Different from other metamodels that are made to explain methods in details, the charts of concepts are to help understanding of the concepts of methods using graphic presentation.

With respect to the charts of concepts, the author introduced the models in details about its primary data types: concept and definitional dependency. A concept of a model is "anything that can be used by software analysts and/or designers to represent or to specify phenomena of the real world or technical phenomena". The definitional dependency defines the dependency relationship between the concepts. The author presented different representations of these two data types, such as root concepts, terminal concepts, and the logical expressions between definitional dependencies. It can be expanded to represent a macro view of a specific methodology. He also presented an overview of the models of UML modelling language by using charts of concepts model to demonstrate its applicability.

However, from my point of view, the usability-related issues are the most critical issues when designing such kind of conceptual models. Therefore, I would have appreciated to see a detailed discussion of e.g. the following aspects in the paper: 
- Why overviews of models are needed, i.e. illustrate any application domains, such as improving reuse process in method construction, and so on?

- The author claimed that "the principal uses of concepts are: verification of models, selection of coherent sub-set in models, creation of customised versions of models, definition of overviews of models, and evaluation of models". However, it remains unclear how the goals are achieved. I would like to see some concrete comparisons between this simple model and other semantic richness model such as GOPRR.

- What are the weaknesses and constraints of such models?

Meanwhile, the author mentioned in the paper, "a complementary study of the semantics of a definitional dependency can conclude to define "A Kind Of" relationship or an inheritance between the two concepts concerned". The fundamental benefits from the charts of Concepts are their simplified representation of the dependence relationship. Being enriched the semantics, it will be back to the same complex situation of ER metamodel or other $\mathrm{OO}$ metamodels and lose its worthy. Moreover, the work of identifying what the "concepts" are seems no different from other approached such as GOPRR. That is to say it is still a hard work.

To summarise, the paper makes a valuable conceptual contribution by proposing the charts of concepts model to ease the understanding of methodologies. On the other hand, the author needs to spend more time motivating the usefulness of this model. 